\title{
Errors of Mean Dynamic Topography and Geostrophic Current Estimates in China's Marginal Seas from GOCE and Satellite Altimetry
}

\author{
SHUANGGEN JIN \\ Department of Geomatics Engineering, Bulent Ecevit University, Zonguldak, Turkey, and Shanghai \\ Astronomical Observatory, Chinese Academy of Sciences, Shanghai, China \\ GUIPING FENG \\ Shanghai Astronomical Observatory, Chinese Academy of Sciences, Shanghai, and \\ University of Chinese Academy of Sciences, Beijing, China \\ OLE ANDERSEN \\ DTU Space, National Space Institute, Technical University of Denmark, Lyngby, Denmark
}

(Manuscript received 16 November 2013, in final form 22 July 2014)

\begin{abstract}
The Gravity Field and Steady-State Ocean Circulation Explorer (GOCE) and satellite altimetry can provide very detailed and accurate estimates of the mean dynamic topography (MDT) and geostrophic currents in China's marginal seas, such as, the newest high-resolution GOCE gravity field model GOCONS-GCF-2-TIM-R4 and the new Centre National d'Etudes Spatiales mean sea surface model MSS_CNES_CLS_11 from satellite altimetry. However, errors and uncertainties of MDT and geostrophic current estimates from satellite observations are not generally quantified. In this paper, errors and uncertainties of MDT and geostrophic current estimates from satellite gravimetry and altimetry are investigated and evaluated in China's marginal seas. The cumulative error in MDT from GOCE is reduced from 22.75 to $9.89 \mathrm{~cm}$ when compared to the Gravity Recovery and Climate Experiment (GRACE) gravity field model ITGGrace2010 results in the region. The errors of the geostrophic currents from GRACE are smaller than from GOCE with the truncation degrees 90 and 120. However, when the truncation degree is higher than 150 , the GRACE mean errors increase rapidly and become significantly larger than the GOCE results. The geostrophic velocities based on GOCE-TIM4 have higher accuracy and spatial resolution, and the mean error is about $12.6 \mathrm{~cm} \mathrm{~s}^{-1}$, which is more consistent with the in situ drifter's results than using GRACE data.
\end{abstract}

\section{Introduction}

China's marginal seas include the South China Sea, East China Sea, Bohai Sea, and Yellow Sea, which connects the Pacific Ocean, Indian Ocean through Taiwan Strait, Luzon Strait, and Malacca Strait, and the Sea of Japan through the Korea Strait (Zhou et al. 1994; Jin et al. 2013). The China's marginal seas have received attention in oceanography research, and one of the important research issues is to understand the patterns of ocean circulation (Liu et al. 2008). The upper-ocean

\footnotetext{
Corresponding author address: Shuanggen Jin, Shanghai Astronomical Observatory, Chinese Academy of Sciences, 80 Nandan Road, Shanghai 200030, China.

E-mail: sgjin@shao.ac.cn; sg.jin@yahoo.com
}

circulation of China's marginal seas is very complex, which is influenced by the monsoon field over the sea, coastal rivers into the sea, the Kuroshio, nonlinear effects of tide, and topography. Therefore, a thorough study and understanding of the upper-ocean circulation in China's marginal seas are very important.

The geostrophic currents are closely related to the mean dynamic topography (MDT), which is defined as the difference between the mean sea surface (MSS) height and the geoid (Feng et al. 2013). Currently, satellite altimetry can monitor sea level change with high accuracy and resolution, so the accuracy of global geoid models is the main limitation in estimating an ocean's MDT and the geostrophic currents (Losch and Schröter 2004; Stammer et al. 2007). Nowadays, the new generation of satellite gravimetry provides a unique opportunity to estimate 
high-precision global geoid models (e.g., Jin et al. 2010, 2011). For example, the European Space Agency (ESA) launched a new Gravity Field and Steady-State Ocean Circulation Explorer (GOCE) satellite in 2009, which carries a highly sensitive gravitational gradiometer to detect fine gravity gradient differences. One of GOCE mission objectives is to determine the $1-2-\mathrm{cm}$ geoid at a spatial resolution of less than $100 \mathrm{~km}$ (Drinkwater et al. 2007). The GOCE mission provides a new opportunity in determining the ocean's MDT with high accuracy and spatial resolution. Here we used the newest gravity field model GO-CONS-GCF-2-TIM-R4 (hereafter GOCE-TIM4), which is a GOCE-only solution based on measurements from November 2009 to June 2012 (approximately 26.5 months of effective data). The GOCETIM4 model is a least squares solution using full normal equations for GPS satellite-to-satellite tracking (SST) and four components of gradiometry (Pail et al. 2011).

Numerous studies on the global and regional MDT and geostrophic currents have been reported using the GOCE data, such as in the Arctic (Farrell et al. 2012), North Atlantic (Bingham et al. 2011), and global and regional oceans (Knudsen et al. 2011; Sánchez-Reales et al. 2012). The Southern Ocean was investigated by Albertella et al. (2012) and Janjić et al. (2012), and the Antarctic Circumpolar Current was studied by Feng et al. (2013). There are two limitations to a detailed ocean circulation determination in China's marginal seas from satellite. First, the errors in the MDT may be larger near the coast than in the deep ocean regions, although satellite altimetry data have been increasingly applied toward coastal oceans in recent years (e.g., Vignudelli et al. 2011; Liu et al. 2012). Second, besides the Kuroshio, multiscale eddies play an important role in ocean circulation variability in China's marginal seas, as seen from both satellite-derived geostrophic currents (e.g., Liu et al. 2008) and in situ observations (e.g., Yuan et al. 1998; Liu et al. 2000). Small errors

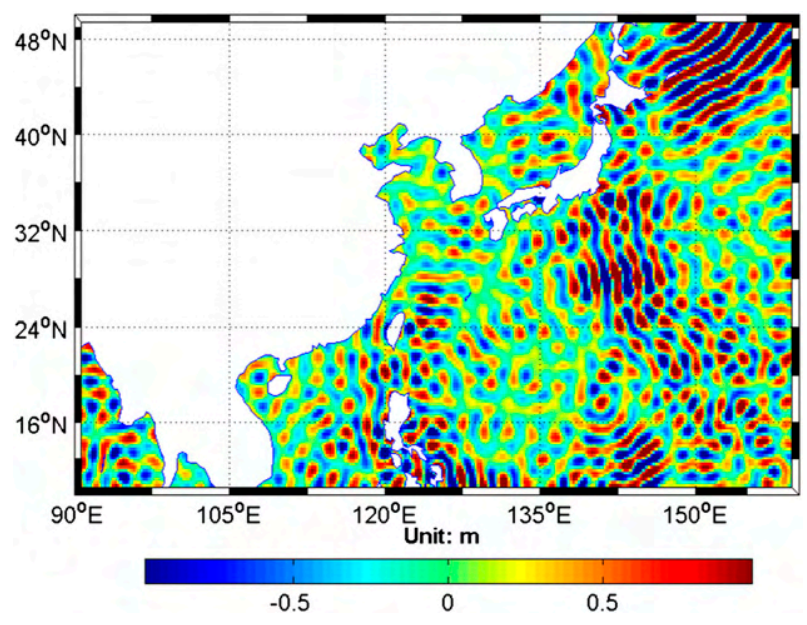

FIG. 1. Difference of geoid between the GOCE (GOCE-TIM4) and GRACE (ITG-Grace2010) in China's marginal seas with up to degree and order 180 .

and uncertainties in geostrophic velocity calculations may affect the interpretation of the ocean circulation patterns. In this paper, the errors and uncertainties of MDT and geostrophic velocities are investigated and analyzed in China's marginal seas based on the newest satellite gravity field models and mean sea surface model. Section 2 presents the observation data and model. Section 3 shows the methods of MDT and geostrophic current error estimates as well as results. The conclusions are given in section 4 .

\section{Observation data and models}

\section{a. Geoid}

The geoid is an equipotential surface, which is most closely coincided with mean sea level. The geoid height can be expressed approximately by spherical harmonic coefficients as follows (Chao and Gross 1987):

$$
N(\vartheta, \lambda, r)=\frac{G M^{t p}}{r \gamma_{0}} \sum_{n=0}^{N \max }\left(\frac{a^{t p}}{r}\right)^{n} \sum_{m=0}^{n}\left(\Delta \overline{\mathrm{C}}_{n m}^{t p} \cos m \lambda+\Delta \overline{\mathrm{S}}_{n m}^{t p} \sin m \lambda\right) \bar{P}_{n m}(\cos \vartheta)
$$

where $(\vartheta, \lambda)$ is the geographic colatitude and longitude, $G M$ is the gravitational constant times the total mass, $r$ is the radial distance of computation point from geocenter, $a$ is the mean radius of the earth, $\gamma_{0}$ is the normal gravity at reference ellipsoid, $\widetilde{P}_{l m}$ are the fully normalized associated Legendre functions of degree $l$ and order $m$, and $\left(\Delta \overline{\mathrm{C}}_{n m}, \Delta \overline{\mathrm{S}}_{n m}\right)$ are the residual coefficients of the spherical harmonic series after subtracting the coefficients of the normal potential from the gravitational potential; superscript $t_{p}$ denotes the fixed values referring to the
Ocean Topography Experiment (TOPEX/Poseidon) reference ellipsoid. Using the gravity field coefficients from GOCE or from the Gravity Recovery and Climate Experiment (GRACE), the geoid can be determined.

Here we compare the geoid derived from the GOCE-TIM4 with the latest GRACE gravity model ITGGrace2010 (Mayer-Gürr et al. 2010). The ITG-Grace2010 is a static gravity field model with spherical harmonic degree and order of up to 180 , which is based on the GRACE-only measurements from August 2002 to August 
2009. Figure 1 shows the difference of geoids between the ITG-Grace2010 and GOCE-TIM4 in China's marginal seas. The difference is up to $0.80 \mathrm{~m}$ with some significant striations in the South China Sea and the North Pacific Ocean.

\section{b. Mean sea surface}

The geodetic MDTs are calculated by subtracting the geoid heights from a MSS. Here we use the mean sea surface model MSS_CNES_CLS_11 provided by Collecte Localisation Satellites (CLS). The model has been computed using 16 years of TOPEX/Poseidon, European Remote Sensing Satellite-2 (ERS-2), Geosat Follow-On (GFO), Jason-1, and Environmental Satellite (Envisat) mean profile and the two 168-day nonrepeat cycle data of the ERS-1 geodetic phase (Hernandez et al. 2000; Hernandez and Schaeffer 2000). The model provides MSS at regular grid with a $1 / 30^{\circ}(2 \mathrm{~min})$ spacing (i.e., $\sim 4 \mathrm{~km}$ ). This model also provides estimation errors from the local

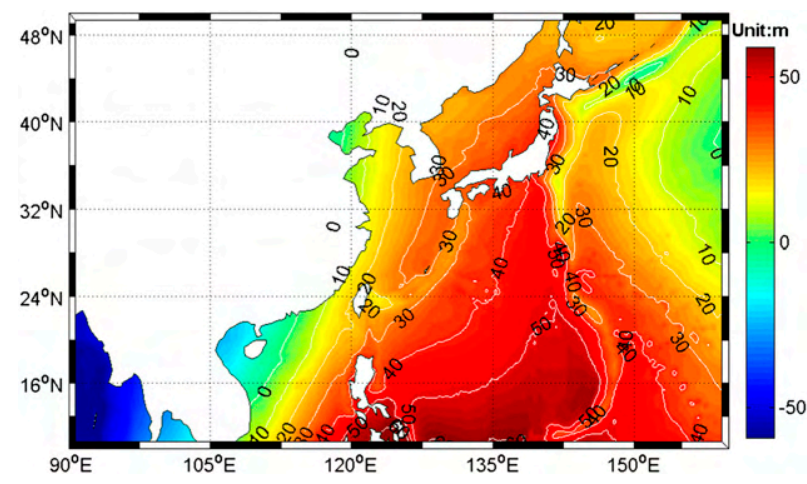

FIG. 2. MSS over China's marginal seas.

inverse technique and calibrated results of crossover point analysis of altimetric data. Figure 2 shows the MSS in China's marginal seas, and Fig. 3 shows the MSS errors in China's marginal seas near the coasts and along the coastlines, such as the Philippine Islands and Japan Islands, where the MSS errors are up to $4 \mathrm{~cm}$.

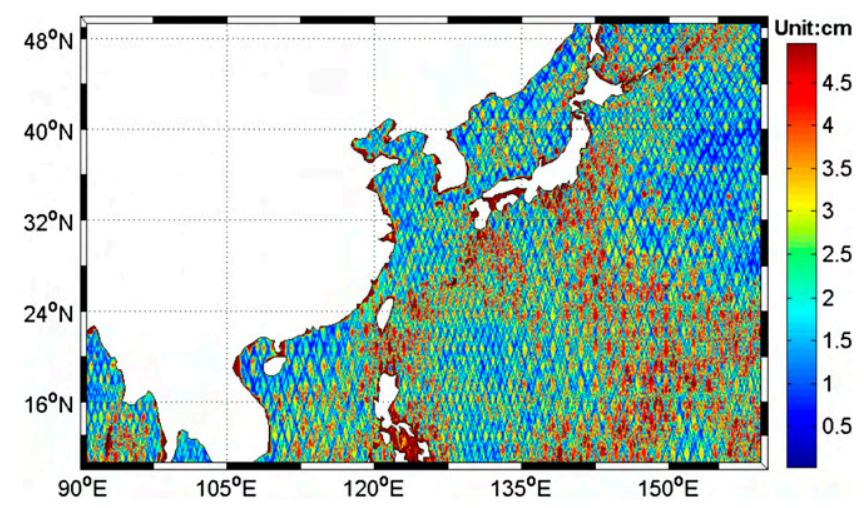

(a) GRACE_N_error

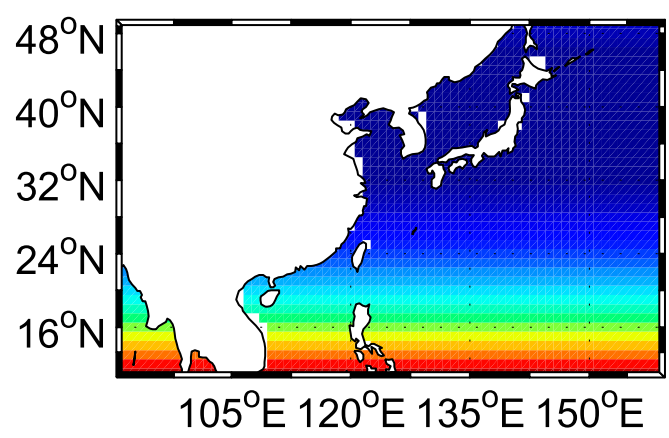

Unit: cm

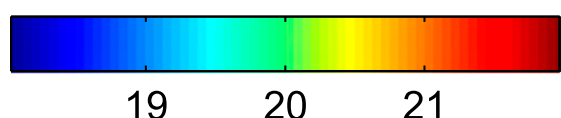

(b) GOCE_N_error

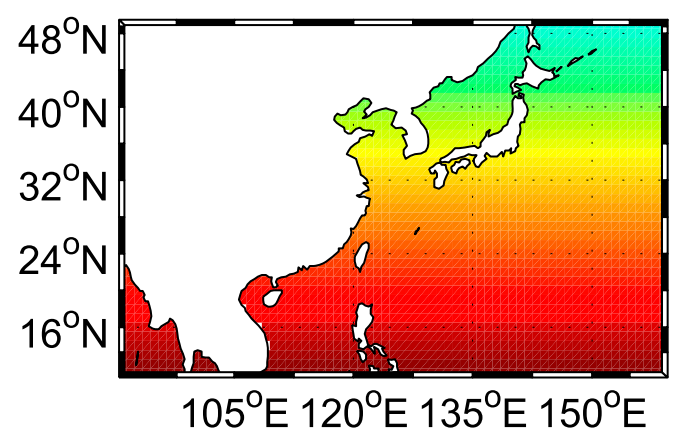

Unit: $\mathbf{c m}$

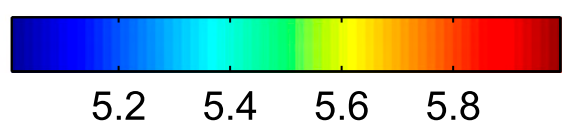

FIG. 3. Errors of the MSS_CNES_CLS_11 in China's marginal seas. (top) Errors of the geoid from different gravity models in China's marginal seas: (a) ITG-Grace2010 and (b) GOCE-TIM4. 
(a) GRACE_MDT

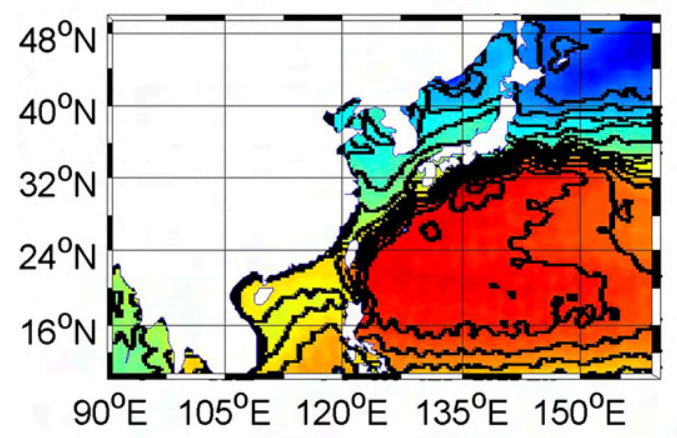

(b) GOCE_MDT

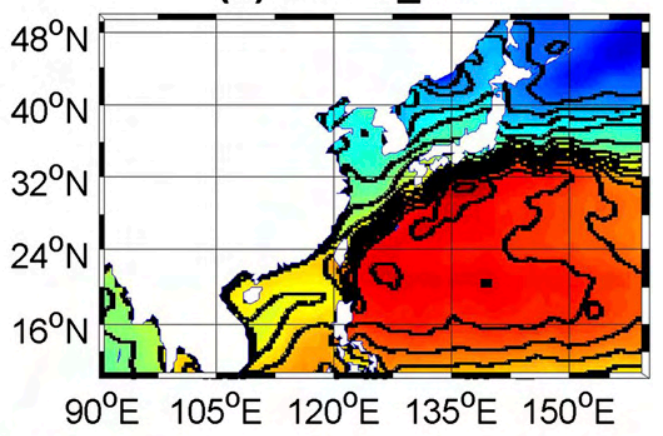

Units: $\mathbf{m}$

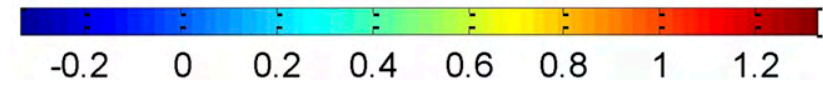

FIG. 4. The MDT in China's marginal seas based on (a) the ITG-Grace2010 gravity model and (b) the GOCE-TIM4 gravity model.

\section{Errors in MDT and geostrophic velocities}

\section{a. Errors in MDT}

The geodetic MDT $(H)$ is defined as the difference between the MSS height $h$ and the geoid height $N$ :

$$
H=h-N \text {. }
$$

To compute a consistent geodetic MDT, the geoid and the MSS are referred to the same coordinate system and reference ellipsoid, while the permanent tide should be treated consistently (Hughes and Bingham 2008; Bingham et al. 2008; Haines et al. 2011). Here, the geoid and MSS are referred to the TOPEX/Poseidon ellipsoid and defined in the mean tide system (no permanent tide effects are removed). The MSS height $h$ can be expanded as a sum of spherical harmonic coefficients as

$$
h(\vartheta, \lambda)=R \sum_{l=0}^{L} \sum_{m=0}^{l}\left(\mathbf{C}_{l m}^{h} \cos m \lambda+S_{l m}^{h} \sin m \lambda\right) \widetilde{P_{l m}}(\cos \vartheta) .
$$

Based on Eqs. (1)-(3), the MDT can be represented as a series of spherical harmonic functions (Albertella et al. 2012; Feng et al. 2013):

$H(\vartheta, \lambda)=R \sum_{l=0}^{L} \sum_{m=0}^{l}\left(\mathbf{C}_{l m}^{H} \cos m \lambda+S_{l m}^{H} \sin m \lambda\right) \widetilde{P_{l m}}(\cos \vartheta)$,

where $\left\{\begin{array}{l}\mathbf{C}_{l m}^{H}=\mathbf{C}_{l m}^{h}-\mathbf{C}_{l m} \\ S_{l m}^{H}=S_{l m}^{h}-S_{l m}\end{array} ;\right.$ the coefficients $\mathbf{C}_{l m}^{H}, S_{l m}^{H}$ are the difference between the filtered coefficients of the spherical harmonic expansion of the MSS $\left(\mathbf{C}_{l m}^{h}, S_{l m}^{h}\right)$; and $\left(\mathbf{C}_{l m}, S_{l m}\right)$ are from the gravity models.

Over land, the MSS_CNES_CLS_11 contains the EIGEN_GRACE_5C mean geoid. To compare ITGGrace2010 with GOCE-TIM4 results, we replace the EIGEN_GRACE_5C mean geoid with the GRACE/ GOCE geoid. In our case the MSS is complemented on land by geoid heights (GRACE/GOCE gravity models):

$$
h^{\text {ext }}=\left\{\begin{array}{ll}
h & \text { (on ocean) } \\
N_{\text {grace } / \text { goce }} & \text { (on land) }
\end{array} .\right.
$$

Then we translate the grid MSS to the spherical harmonic coefficients (Albertella and Rummel 2009; Feng et al. 2013). Since the spatial resolution of MSS is significantly higher than the gravity models, these short-scale features involved in the MDT should be removed by filtering to make sure that the geodetic MDTs are consistent with the spatial resolution of the gravity field.

Here we apply a Gauss filter with a half-weight radius $r$ of the filter that is related to the harmonic degree $L$ of the spectrum $(r=20000 / L)$ (Jekeli 1981; Wahr et al. 1998). Figure 4 shows the MDT computed from the gravity model ITG-Grace2010 (Fig. 4a) and MDT computed from the GOCE-TIM4 (Fig. 4b). The filtering is up degree $L=180$. When compared with each other, the GRACE MDT results have some errors, indicated by contour lines that are not very smooth.

The error variance-covariance matrix $\mathbf{C}_{H H}$ of $H$ from Eq. (4) is expressed as 
(a) GRACE_MDT_error

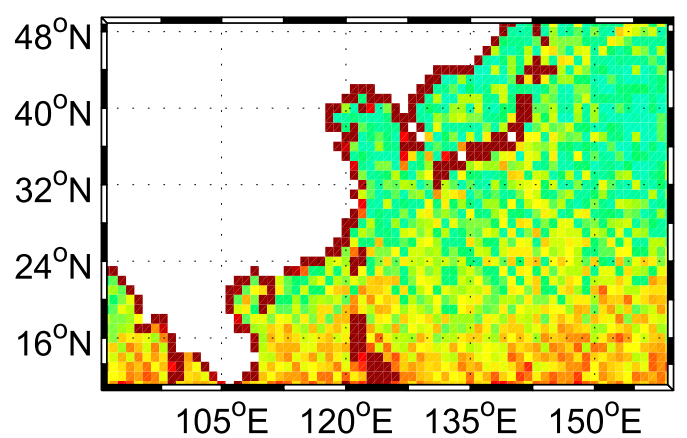

Unit: $\mathbf{c m}$

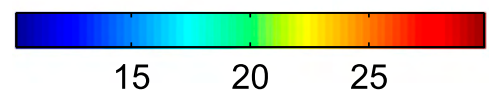

(b) GOCE_MDT_error

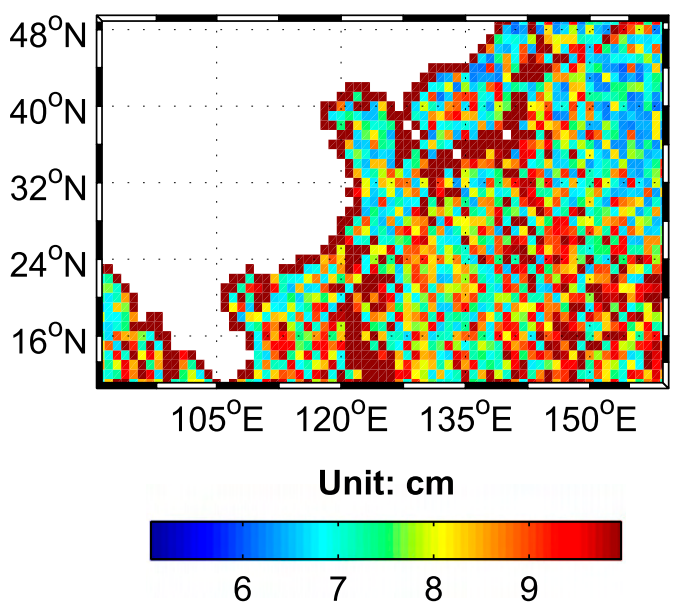

FIG. 5. The propagated errors from the harmonic coefficients to the MDT in China's marginal seas based on (a) the ITG-Grace2010 gravity model and (b) the GOCE-TIM4 gravity model.

$$
\mathbf{C}_{H H}=\mathbf{C}_{h h}+\mathbf{C}_{N N}-2 \mathbf{C}_{h N}
$$

where $\mathbf{C}_{h h}$ is the variance-covariance matrix of the MSS $(h), \mathbf{C}_{N N}$ is the variance-covariance matrix of $N$ and $\mathbf{C}_{h N}$ is the covariance matrix between $h$ and $N$. The correlations between $N$ and $h$ are assumed to be zero, since both of these are derived from independent measurements. By simplification, we can get

$$
\mathbf{C}_{H H}=\mathbf{C}_{h h}+\mathbf{C}_{N N} .
$$

For the geoid errors, we confine the $\mathbf{C}_{N N}$ matrix to its diagonal part-that is, to the error variancesneglecting the correlations between the spherical

$$
\left\{\begin{array}{l}
\sigma \mathbf{C}_{l m}^{h} \\
\sigma S_{l m}^{h}
\end{array}\right\}=\frac{1}{4 \pi R} \int_{0}^{2 \pi} d \lambda \int_{0}^{\pi} \sin \vartheta d \vartheta \cdot \sigma h(\vartheta, \lambda) P_{l m}(\cos \vartheta)\left\{\begin{array}{c}
\cos (m \lambda) \\
\sin (m \lambda)
\end{array}\right\} .
$$

The MSS error harmonics coefficients should be filtered, and the Gaussian filter is applied to MSS and $N$ when combined. We can use the following function to consider the filter's influence: $\left(\bar{\sigma} \mathbf{C}_{l m}\right)^{2}=$ $\left(W_{l}\right)^{2}\left(\sigma \mathbf{C}_{l m}\right)^{2},\left(\bar{\sigma} S_{l m}\right)^{2}=\left(W_{l}\right)^{2}\left(\sigma S_{l m}\right)^{2}$, where $W_{l}$ is the Gaussian filter. From error variances of the satellite gravity field model's harmonic coefficients and error variances of MSS estimate, we can obtain the informal errors of the geodetic MDTs based on Eq. (6). As shown in Fig. 5, the total mean uncertainty of ITGGrace2010 MDT is around $22.75 \mathrm{~cm}$, while the uncertainty of the MDT using GOCE-TIM4 is about $9.89 \mathrm{~cm}$ to the same degree and order of 180 . The harmonic coefficients of the gravity model. For the MSS errors, we should translate the grid MSS errors into harmonics coefficients while the truncation degree should be the same as gravity models. The MSS errors can be described as

$$
\begin{aligned}
\sigma h(\vartheta, \lambda)= & R \sum_{l=0}^{L} \sum_{m=0}^{l}\left(\sigma \mathbf{C}_{l m}^{h} \cos m \lambda\right. \\
& \left.+\sigma S_{l m}^{h} \sin m \lambda\right) \widetilde{P_{l m}}(\cos \vartheta) .
\end{aligned}
$$

Using the grid MSS error $\sigma h(\vartheta, \lambda)$, we can calculate $\left(\sigma \mathbf{C}_{l m}^{h}, \sigma S_{l m}^{h}\right)$ in the following function

errors along the coastlines and near the islands are larger, mainly because of the sparse satellite altimetric data in these regions. To further analyze the uncertainties of these two geodetic MDTs, we conducted a statistical analysis. Figure 6a shows that most RMSs based on ITG-Grace2010 MDT are located in the 16$28-\mathrm{cm}$ range, with almost $51 \%$ in the $16-20-\mathrm{cm}$ range, $37 \%$ in the $20-24-\mathrm{cm}$ range, and $5 \%$ in the $24-28-\mathrm{cm}$ range. The RMSs with errors larger than $28 \mathrm{~cm}$ are focused along the coastlines. In Fig. 6b, most RMSs based on GOCE-TIM4 MDT are located in the 4-12-cm range with almost $46 \%$ in the $4-8-\mathrm{cm}$ range and $43 \%$ in the $8-12-\mathrm{cm}$ range (see Table 1). Therefore, the RMS of 
(a) RMS probability density distribution based on GRACE_MDT

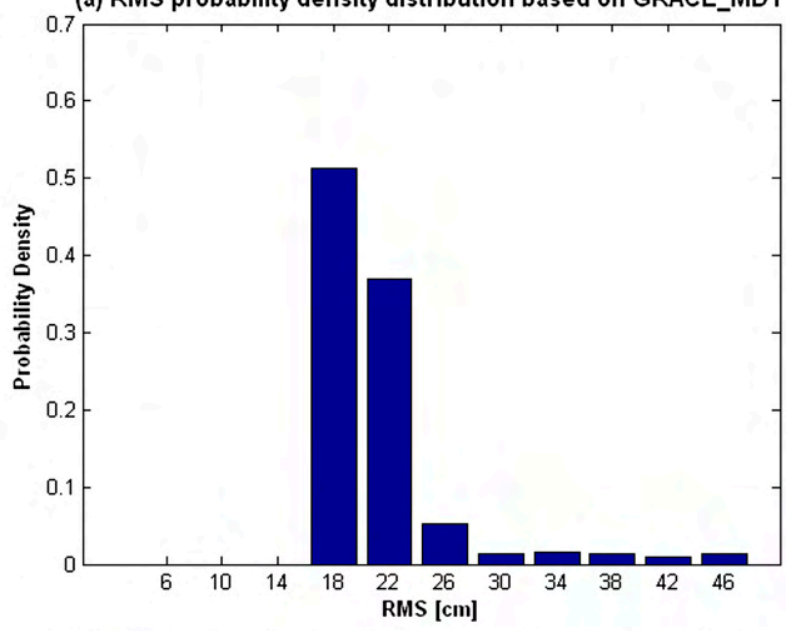

(b) RMS probability density distribution based on GOCE_MDT

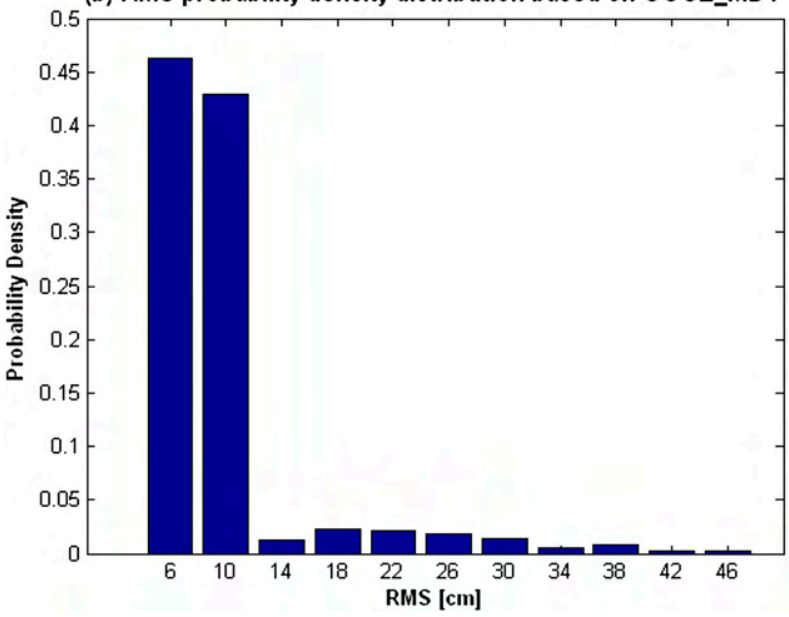

FIG. 6. RMS probability density distribution based on (a) ITG-Grace2010 MDT and (b) GOCE-TIM4 MDT.

the GOCE-TIM4 MDT is significantly smaller than the ITG-Grace2010 results at each grid point, especially along the coastlines and near the islands. The percentages of geoid errors in the geodetic MDT errors are further given. As shown in Fig. 7, for ITG-Grace2010 MDT, the geoid error is the main source in China's marginal seas, and the percentage is up to $90 \%$ in most regions. Along the coastlines and inlands, the percentage is much smaller by nearly $50 \%$. The geoid error accounts for $85.35 \%$ of the ITG-Grace2010 MDT errors. For the GOCE-TIM4 MDT, the phenomenon is opposite. In the East China Sea, South China Sea, and North Pacific Ocean, the percentage is between $55 \%$ and $70 \%$. The geoid error accounts for $67.71 \%$ in the GOCETIM4 MDT errors. Therefore, the GOCE-TIM4derived MDT is significantly more accurate than the ITG-Grace2010 MDT results. And the percentage of the GOCE-TIM4 geoid error in the MDT errors is much smaller than the ITG-Grace2010 results.

We also calculated the errors of the geodetic MDTs as a function of truncation degrees. Figure 8 shows the cumulative error of geodetic MDTs based on ITG-Grace2010 and GOCE-TIM4 in China's marginal seas for truncation degree of up to $L=90,120$, 150 , and 180 . When the truncation degrees are 90 and 120, the errors of ITG-Grace2010 MDT are less than the GOCE-TIM4 MDT errors. However, the GOCE-TIM4 accuracy is improved for degrees of higher than 150 when compared to ITG-Grace2010. The MDT's errors show that the ITG-Grace2010 MDT is precise in the lower truncation degree (less than 120), while GOCE-TIM4 MDT can capture the shorter scale information very accurately (higher than degree 150).

\section{b. Errors in geostrophic velocities}

The surface geostrophic currents are directly related to the gradient of MDT. The surface geostrophic velocities $\left(u_{s}, v_{s}\right)$ can be calculated as

$$
\begin{aligned}
& u_{s}=-\frac{g}{f} \frac{\partial H}{\partial y}=-\frac{g}{f} \frac{\partial H}{R \partial \vartheta} \\
& v_{s}=\frac{g}{f} \frac{\partial H}{\partial x}=\frac{g}{f} \frac{\partial H}{R \sin \vartheta \partial \lambda}
\end{aligned}
$$

Based on Eq. (4), the surface velocities can be expressed in spherical harmonic coefficients as

$$
\begin{aligned}
& u_{s}=-\frac{g}{f} \frac{1}{R} \sum_{l=0}^{L} \sum_{m=0}^{l} R\left(\mathbf{C}_{l m}^{H} \cos m \lambda+S_{l m}^{H} \sin m \lambda\right) \widetilde{P_{l m}^{\prime}}(\cos \vartheta) \\
& v_{s}=\frac{g}{f} \frac{1}{R \sin \vartheta} \sum_{l=0}^{L} \sum_{m=0}^{l} m R\left(-\mathbf{C}_{l m}^{H} \sin m \lambda+S_{l m}^{H} \cos m \lambda\right) \widetilde{P_{l m}}(\cos \vartheta),
\end{aligned}
$$

where $g$ is the gravitational acceleration, $f=2 \Omega \cos \vartheta$ is the Coriolis parameter depending on colatitude $\vartheta, \Omega$ is the angular velocity of the earth, $R$ is the mean earth radius, and $\widetilde{P^{\prime}}$ is the first derivative with respect to $\vartheta$ of the associated Legendre function $\widehat{P_{l m}}$. For the surface geostrophic currents vectors, the direction is $\mathbf{A}=\arctan \left(u_{s} / v_{s}\right)$ 
TABLE 1. Statistical results (\%) of RMS probability density distribution based on ITG-Grace2010 MDT and GOCE-TIM4 MDT.

\begin{tabular}{llllllllllll}
\hline \hline & $4-8$ & $8-12$ & $12-16$ & $16-20$ & $20-24$ & $24-28$ & $28-32$ & $32-36$ & $36-40$ & $40-44$ & $>44$ \\
\hline GRACE & 0 & 0 & 0 & 0.51 & 0.37 & 0.05 & 0.01 & 0.02 & 0.02 & 0.01 & 0.01 \\
GOCE & 0.46 & 0.43 & 0.01 & 0.02 & 0.02 & 0.02 & 0.02 & 0.01 & 0.01 & 0 & 0 \\
\hline
\end{tabular}

and the length is $\mathbf{V}=\sqrt{u_{s}^{2}+v_{s}^{2}}$ (Elema 1993). Based on Eq. (10), we can calculate the geostrophic currents velocities from geodetic MDTs.

To assess the geostrophic currents from geodetic MDTs, a comparison with independent drifter data was performed. Here we use the drifter-derived global nearsurface currents (Lumpkin and Johnson 2013). The near-surface currents covering the areas from $73^{\circ} \mathrm{S}$ to $85^{\circ} \mathrm{N}$, at annual mean and one-half degree resolution, are derived from satellite-tracked drifters of the Global Drifter Program (Niiler 2001; Lumpkin and Pazos 2007). Since near-surface currents include geostrophic currents, Ekman currents, and high-frequency ageostrophic currents, we have to remove the influence of Ekman currents in order to perform a consistent comparison with the geostrophic currents from geodetic MDTs. Here the wind stress and the local Coriolis parameter were used to estimate the Ekman component (Ralph and Niiler 1999). In this study, the wind stress was calculated from the surface winds using a constant neutral drag coefficient of $1.2 \times 10^{-3}$. The daily winds were from the National Centers for Environmental PredictionNational Center for Atmospheric Research (NCEPNCAR) reanalysis (Niiler and Paduan 1995; Pazan and Niiler 2001).

Figures $9 a-d$ show the mean geostrophic velocities in China's marginal seas estimated from ITG-Grace2010, GOCE-TIM4, drifters' measurements, and the CNES CLS_09 (Rio et al. 2011), respectively. The general

\section{(a) GRACE_error}

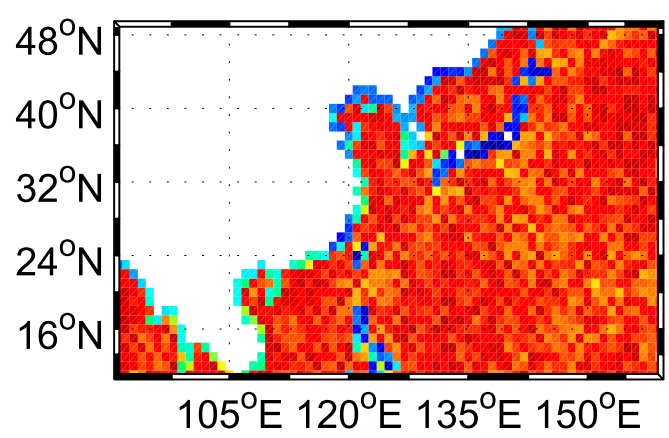

circulation pattern of the Kuroshio is clearly seen from the GRACE, GOCE, drifters' measurements, and CNES_CLS_09. North Equatorial Currents between $10^{\circ}$ and $17^{\circ} \mathrm{N}$ turn to the north in the Philippines and form the Kuroshio. The Kuroshio branches off into two subbranches in the Luzon Strait. One portion enters the South China Sea and the other one continues to flow northeastward from southeastern Taiwan and along the eastern coast of Japan following the continental slope. At about $35^{\circ} \mathrm{N}, 140^{\circ} \mathrm{E}$ near the coast of Japan, the Kuroshio leaves the boundary, flowing east into the North Pacific as the Kuroshio Extension.

Some details are different in the four maps. Along the coastlines, the GRACE results are seen to be noisier, especially in the Bohai Sea and the Taiwan Strait. For the North Equatorial Currents, the GRACE results are unordered, especially in the directions of the currents, while the GOCE results have a quite close agreement with the drifters' results. In Table 2 we give the RMS and correlation coefficients between GRACE, GOCE, and the drifters' results in China's marginal seas. In terms of total velocities, the GOCE RMS is just $16.1 \mathrm{~cm} \mathrm{~s}^{-1}$, while the GRACE RMS is $18.20 \mathrm{~cm} \mathrm{~s}^{-1}$. In the eastern direction, the GOCE RMS is $14.0 \mathrm{~cm} \mathrm{~s}^{-1}$, while GRACE RMS is $15.3 \mathrm{~cm} \mathrm{~s}^{-1}$. In the northern direction, the GOCE RMS is $15.18 \mathrm{~cm} \mathrm{~s}^{-1}$, while GRACE RMS is $16.0 \mathrm{~cm} \mathrm{~s}^{-1}$. The correlation coefficients of the GRACE results are 0.40 in total velocities, 0.60 in the eastern direction, and 0.21 in the northern direction, while the correlation

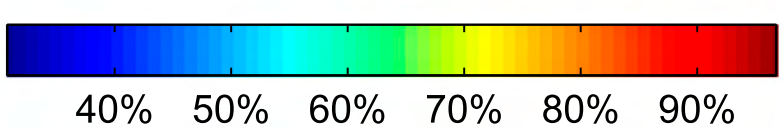

FIG. 7. The geoid error accounted for in the geodetic MDT errors in China's marginal seas based on (a) ITG-Grace2010 and (b) GOCE-TIM4. 

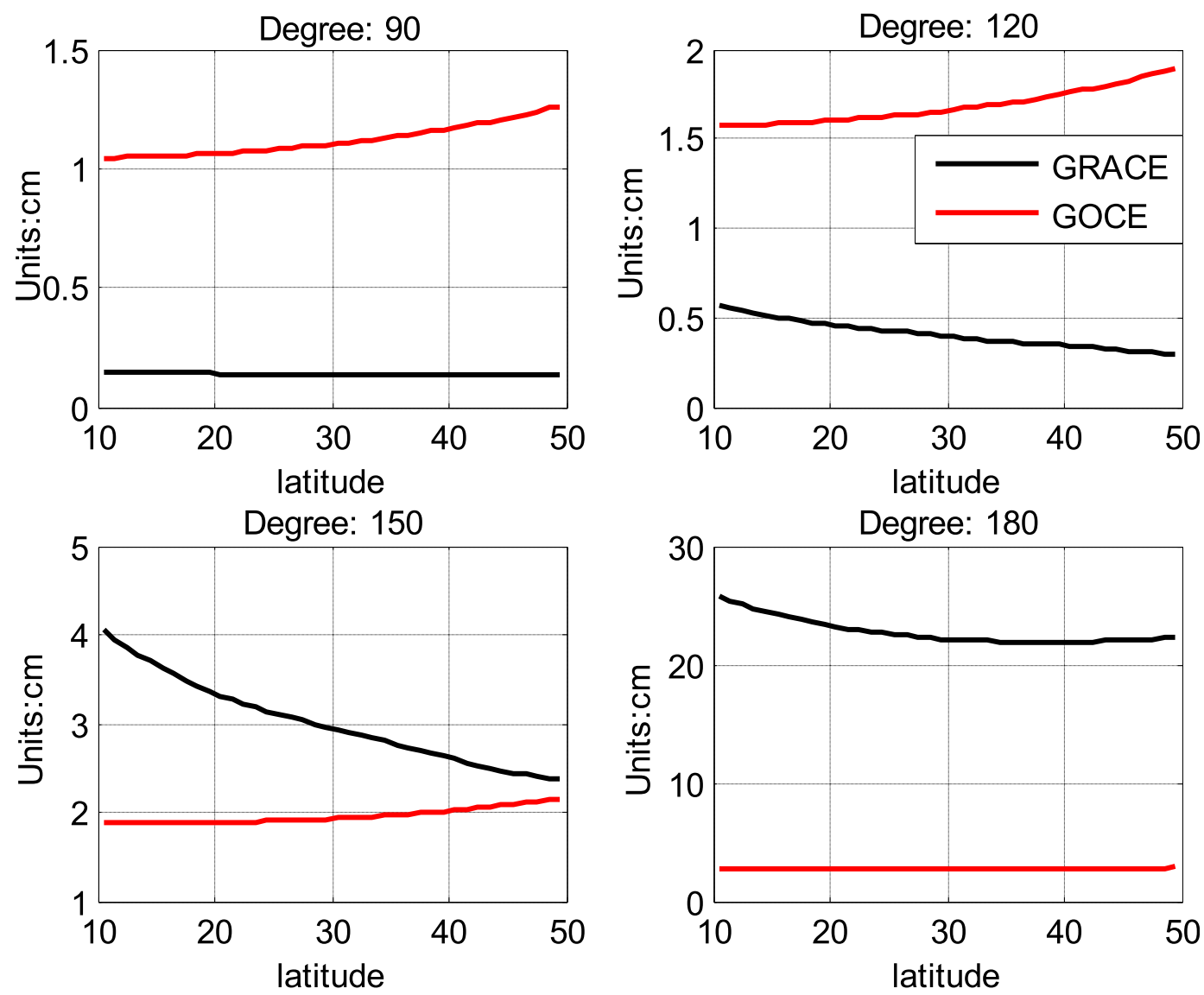

FIG. 8. The cumulative error in geodetic MDTs based on ITG-Grace2010 and GOCE-TIM4 in China's marginal seas for degrees of up to $L=90,120,150$, and 180 .

coefficients of the GOCE results are 0.73 in total velocities, 0.65 in the eastern direction, and 0.26 in the northern direction, much closer to drifters' results than the GRACE in China's marginal seas. We can see clearly that the CNES-CLS_09 result is smaller than the drifters' results (Fig. 9d). We also calculate the RMS and correlation coefficients between CNES_CLS_09 and the drifters' results (Table 2), and the RMS of
CNES_CLS_09 is almost located between the GOCE and GRACE results in China's marginal seas.

The accuracy of the surface geostrophic velocity fields depends on the accuracy of the spherical coefficients of the geodetic MDTs as well as omission errors for the higher-order spherical harmonic constituents omitted in the geodetic MDT. Based on Eq. (10), the errors of geostrophic velocities can be described as

$$
\begin{aligned}
& \left.\left(\sigma u_{s}\right)^{2}=\left(\frac{g}{f}\right)^{2} \frac{1}{R^{2}} \sum_{l=0}^{L} \sum_{m=0}^{l} R^{2}\left[\left(\sigma \mathbf{C}_{l m}^{H}\right)^{2}(\cos m \lambda)^{2}+\left(\sigma S_{l m}^{H}\right)^{2}(\sin m \lambda)^{2}\right] \widetilde{P_{l m}^{\prime}}(\cos \vartheta)\right]^{2} \\
& \left.\left(\sigma v_{s}\right)^{2}=\left(\frac{g}{f}\right)^{2} \frac{1}{R^{2}(\sin \vartheta)^{2}} \sum_{l=0}^{L} \sum_{m=0}^{l} m^{2} R^{2}\left[\left(\sigma \mathbf{C}_{l m}^{H}\right)^{2}(\cos m \lambda)^{2}+\left(\sigma S_{l m}^{H}\right)^{2}(\sin m \lambda)^{2}\right] \widetilde{P_{l m}}(\cos \vartheta)\right]^{2}
\end{aligned}
$$

We can obtain the error coefficients $\left(\sigma \mathbf{C}_{l m}^{H}, \sigma \mathbf{S}_{l m}^{H}\right)$ of MDT. Therefore, we can calculate the errors of geostrophic velocities based on Eq. (9). We also calculated the errors of the geostrophic surface velocity fields based on GRACE and GOCE models as a function of truncation degree. Figure 10 shows the cumulative error of the geostrophic velocities to degrees of up to $L=90$, 120,150 , and 180. The GRACE errors of geostrophic currents are smaller than the GOCE results for truncation degrees of 90 and 120, which indicate that the 

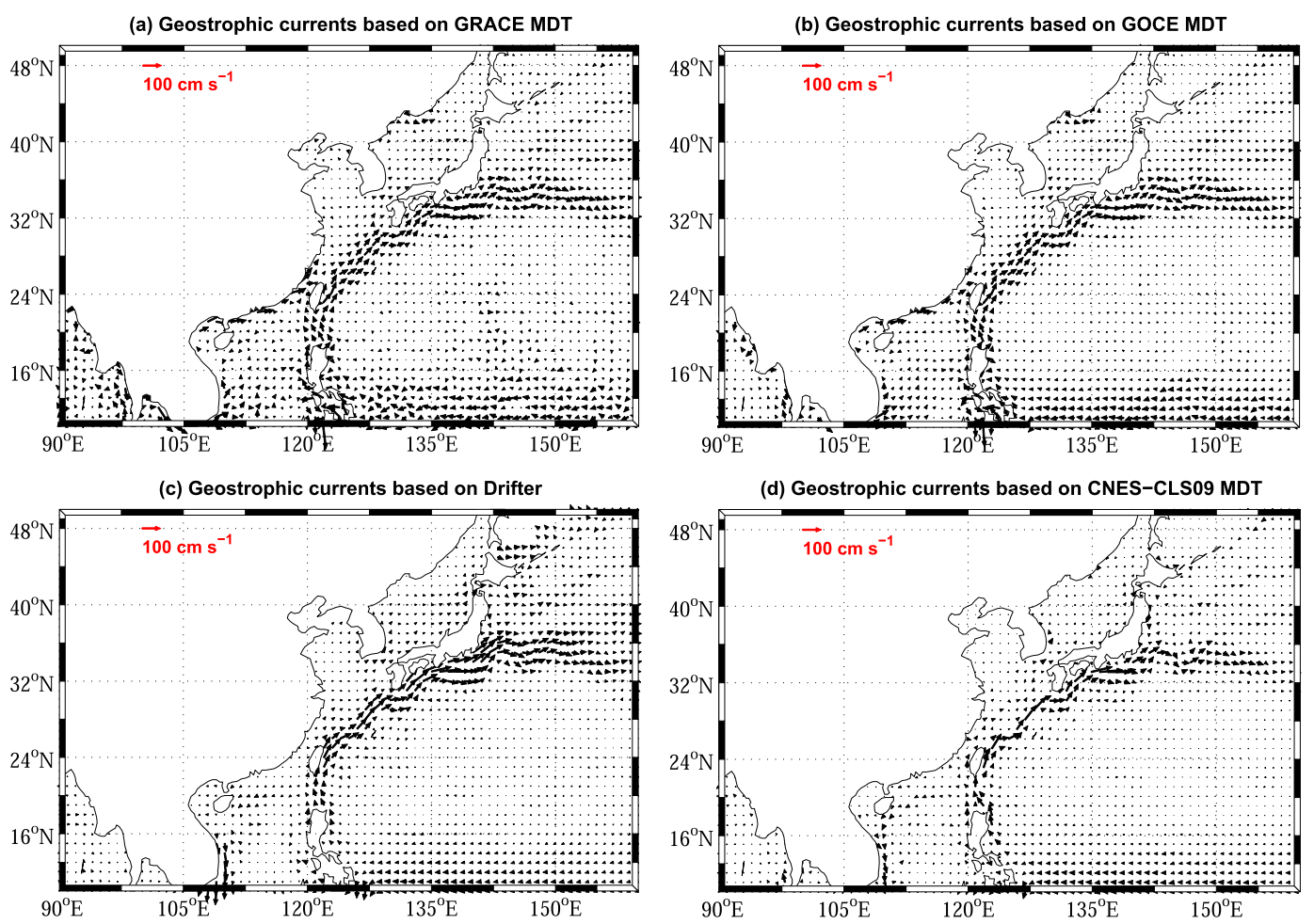

FIG. 9. Geostrophic velocities in China's marginal seas from (a) GRACE geoid (ITG-Grace2010), (b) GOCE geoid (GOCE-TIM4), (c) drifters' measurements, and (d) CNES-CLS09 MDT.

accuracy of ITG-Grace2010 is better than GOCE-TIM4 for the low degree (Figs. 10b,c). However, when the truncation degrees are 150, the GRACE mean errors rapidly increase to $9.83 \mathrm{~cm} \mathrm{~s}^{-1}$ and become larger than the GOCE results. The mean errors of the GRACE results increase from 9.8 to $40.1 \mathrm{~cm} \mathrm{~s}^{-1}$ for a truncation degree of 180 . The total mean error from the ITGGrace 2010 model is around $40.1 \mathrm{~cm} \mathrm{~s}^{-1}$, while the mean RMS error from GOCE-TIM4 is about $9.7 \mathrm{~cm} \mathrm{~s}^{-1}$. The mean error in the eastern direction from the ITGGrace2010 model is around $30.3 \mathrm{~cm} \mathrm{~s}^{-1}$, while the GOCE-TIM4 result is about $7.8 \mathrm{~cm} \mathrm{~s}^{-1}$. Comparing Fig. 10a with Fig. 10b, there is only a little difference at the truncation degree of 150 , which means that the east components of ITG-Grace 2010 are almost the similar to the GOCE-TIM4 results. The northern direction's mean error based on the ITG-Grace 2010 model is around $29.1 \mathrm{~cm} \mathrm{~s}^{-1}$, while the GOCE-TIM4 result is about $5.6 \mathrm{~cm} \mathrm{~s}^{-1}$ (Fig. 10c). For the northern direction's mean error, the GRACE mean errors are larger than the GOCE-TIM4 results when the truncation degree is 120. In Fig. 10, we can find that when it is closer to the equator, the errors of geostrophic velocities are larger for both GRACE and GOCE results. The reason is that when we calculate the errors, the latitude is close to the equator, the Coriolis parameter is close to 0 , and the larger errors of geostrophic velocities will be obtained.

\section{Conclusions}

In this paper, errors and uncertainties of MDT and geostrophic currents in China's marginal seas have been investigated and analyzed. For the MDT, the total mean RMS of ITG-Grace2010 MDT is around $22.75 \mathrm{~cm}$, with almost $51 \%$ of the RMS in the $16-20-\mathrm{cm}$ range and $37 \%$ in the 20-24-cm range, while the RMS of the GOCETIM4 MDT is about $9.89 \mathrm{~cm}$, and almost $46 \%$ falls in the $4-8-\mathrm{cm}$ range and $43 \%$ in the $8-12-\mathrm{cm}$ range. The RMS of the GOCE-TIM4 MDT is significantly smaller than the ITG-Grace2010 results at each grid in China's marginal seas, especially near and along the coastlines

TABLE 2. Comparison of geostrophic current velocities from GRACE and GOCE with the drifters' results in China's marginal seas; corr $=$ correlation coefficients.

\begin{tabular}{|c|c|c|c|c|c|c|}
\hline & \multicolumn{2}{|c|}{$V$} & \multicolumn{2}{|c|}{$u_{s}$} & \multicolumn{2}{|c|}{$v_{s}$} \\
\hline & RMS & Corr & RMS & Corr & RMS & Corr \\
\hline GRACE & 18.2 & 0.40 & 15.3 & 0.60 & 16.0 & 0.21 \\
\hline GOCE & 16.1 & 0.43 & 14.0 & 0.65 & 15.2 & 0.26 \\
\hline CNES-CLS09 & 17.4 & 0.42 & 14.9 & 0.59 & 16.0 & 0.25 \\
\hline
\end{tabular}




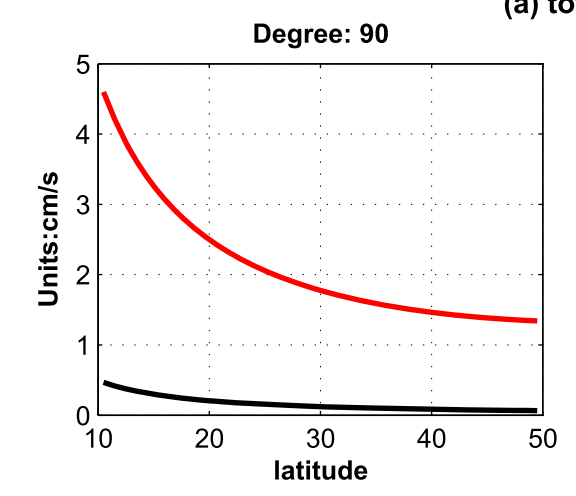

(a) total velocity
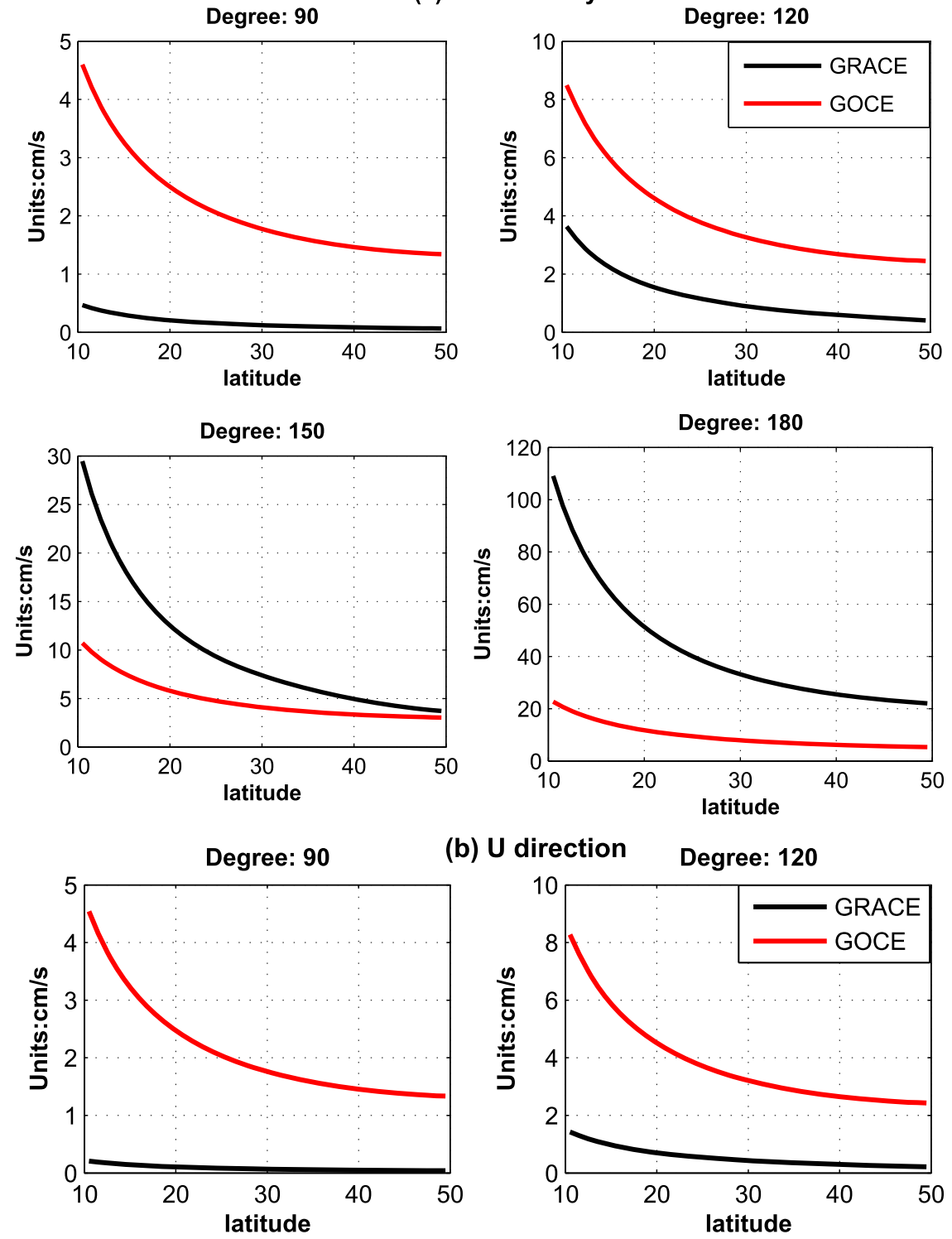

(b) U direction Degree: 120
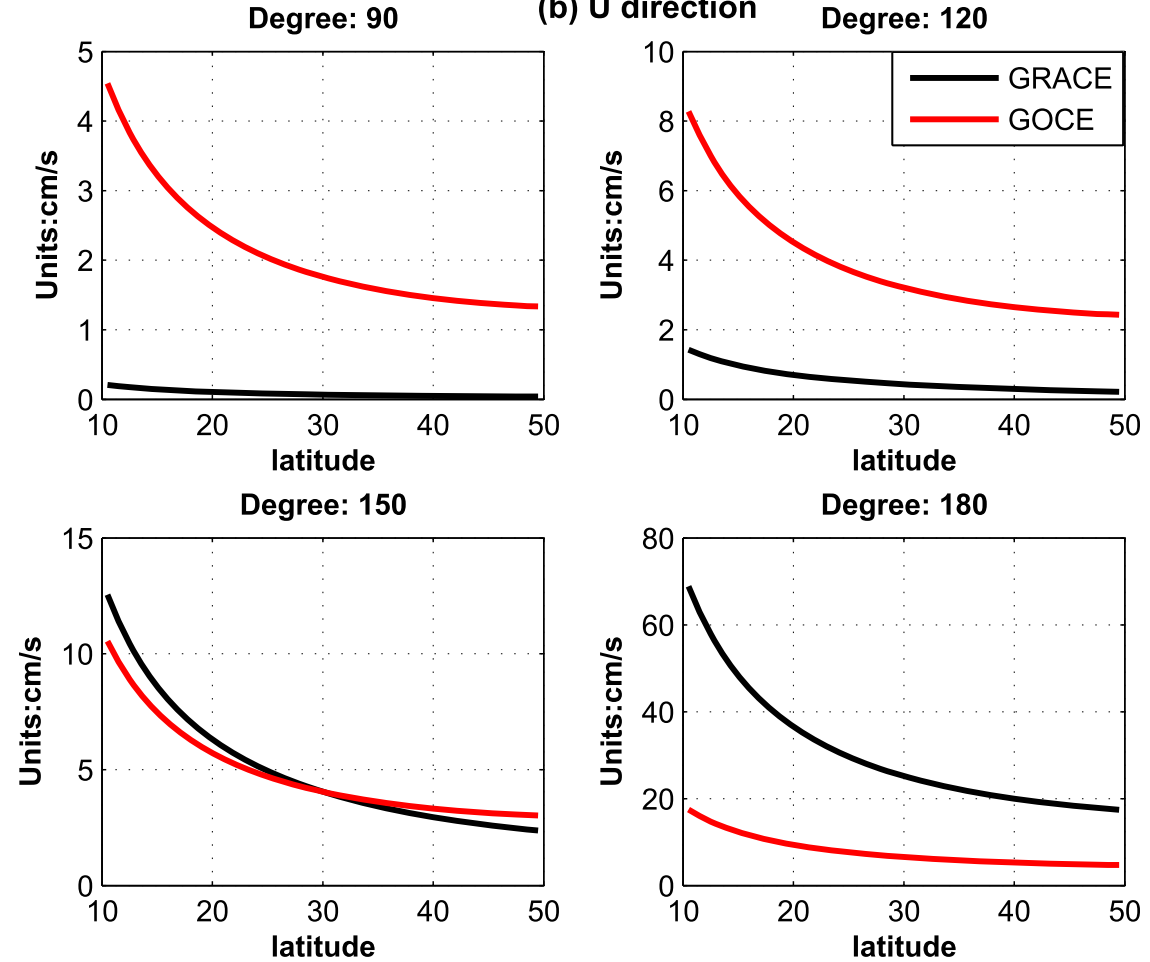

FIG. 10. Cumulative errors in (a) total velocity, (b) eastern direction velocity, (c) and northern direction velocity in China's marginal seas with degrees of up to $L=90,120,150$ and 180 . 


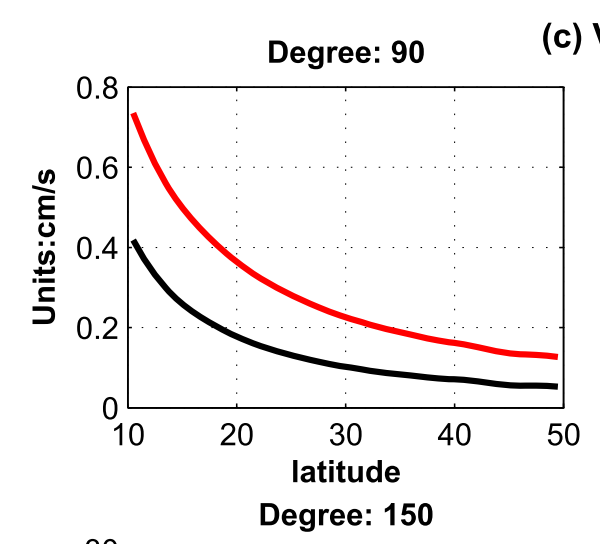

(c) V direction
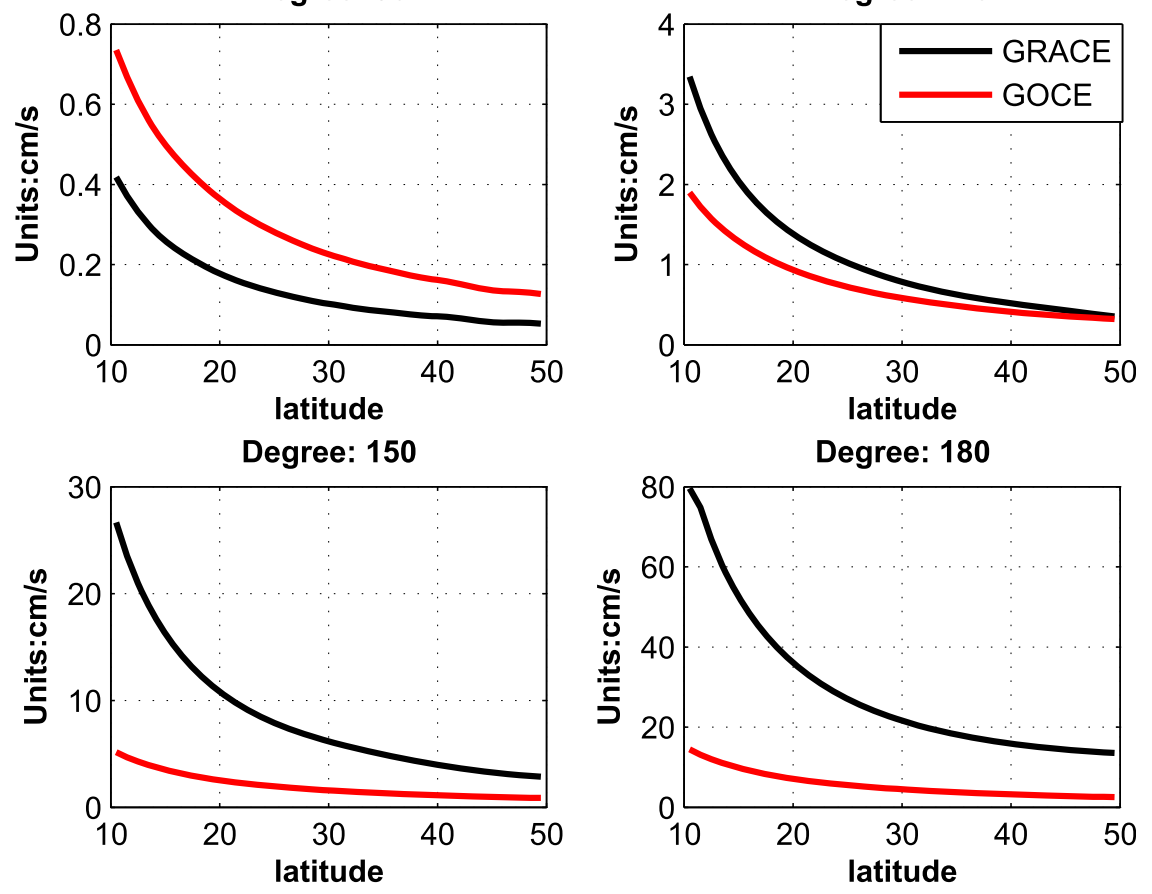

FIG. 10. (Continued)

and near the islands. The GRACE geoid error accounts for $85.35 \%$ of the ITG-Grace2010 MDT errors, while the GOCE geoid error accounts for $67.71 \%$ in the GOCE-TIM4 MDT errors. The errors of the geostrophic currents from GRACE are smaller than the GOCE results for truncation degrees 90 and 120 . However, when the truncation degree is higher than 150 , the GRACE mean errors increase rapidly and become larger than the GOCE results. The total mean error from the ITG-Grace2010 model is around $40.1 \mathrm{~cm} \mathrm{~s}^{-1}$, while the mean error of the geostrophic surface velocities based on GOCE-TIM4 is about $12.6 \mathrm{~cm} \mathrm{~s}^{-1}$. The GOCE results are also much closer to drifters' results than the GRACE in China's marginal seas, indicating that GOCE provides accurate details and high accuracy of geostrophic currents estimate in the China's marginal seas.

Acknowledgments. This research is supported by the Main Direction Project of the Chinese Academy of Sciences (Grant KJCX2-EW-T03), the Shanghai Science and Technology Commission Project (Grant 12DZ2273300), and the National Natural Science Foundation of China (NSFC) Project (Grants 11173050 and 11373059). We are grateful to ESA for providing the GOCE gravity data and to AVISO for providing the ocean altimetry data as well as to the Scientific Research Project Office of Bulent Ecevit University for support.

\section{REFERENCES}

Albertella, A., and R. Rummel, 2009: On the spectral consistency of the altimetric ocean and geoid surface: A one-dimensional example. J. Geod., 83, 805-815, doi:10.1007/s00190-008-0299-5.

—, R. Savcenko, T. Janjić, R. Rummel, W. Bosch, and J. Schröter, 2012: High resolution dynamic ocean topography in the Southern Ocean from GOCE. Geophys. J. Int., 190, 922930, doi:10.1111/j.1365-246X.2012.05531.x.

Bingham, R. J., K. Haines, and C. W. Hughes, 2008: Calculating the ocean's mean dynamic topography from a mean sea surface and a geoid. J. Atmos. Oceanic Technol., 25, 1808-1822, doi:10.1175/ 2008JTECHO568.1.

—, P. Knudsen, O. Andersen, and R. Pail, 2011: An initial estimate of the North Atlantic steady-state geostrophic circulation from GOCE. Geophys. Res. Lett., 38, L01606, doi:10.1029/ 2010 GL045633.

Chao, B. F., and R. S. Gross, 1987: Changes in the Earth's rotation and low-degree gravitational field induced by earthquakes. Geophys. J. Int., 91, 569-596, doi:10.1111/j.1365-246X.1987.tb01659.x.

Drinkwater, M. R., R. Haagmans, D. Muzi, A. Popescu, R. Floberghagen, M. Kern, and M. Fehringer, 2007: The GOCE gravity mission: ESA's First Core Earth Explorer. Proceedings of the 3rd International GOCE User Workshop, K. Fletcher, Ed., ESA Publ. ESA SP-627, 1-8.

Elema, I. A., 1993: Influence of geoid model uncertainty on the determination of the ocean circulation with satellite altimetry. M.S. thesis, Dept. of Geodesy, Delft University of Technology, 109 pp.

Farrell, S. L., D. McAdoo, S. W. Laxon, H. J. Zwally, D. Yi, A. Ridout, and K. Giles, 2012: Mean dynamic topography of the Arctic Ocean. Geophys. Res. Lett., 39, L01601, doi:10.1029/ 2011 GL050052. 
Feng, G. P., S. G. Jin, and J. Sánchez-Reales, 2013: Antarctic circumpolar current from satellite gravimetric models ITGGRACE2010, GOCE-TIM3 and satellite altimetry. J. Geodyn., 72, 72-80, doi:10.1016/j.jog.2013.08.005.

Haines, K., J. A. Johannessen, P. Knudsen, D. Lea, M. H. Rio, L. Bertino, F. Davidson, and F. Hernandez, 2011: An ocean modelling and assimilation guide to using GOCE geoid products. Ocean Sci., 7, 151-164, doi:10.5194/os-7-151-2011.

Hernandez, F., and P. Schaeffer, 2000: Altimetric mean sea surfaces and gravity anomaly maps inter-comparisons. AVISO Rep. AVI-NT-011-5242-CLS, 48 pp.

_, M.-H. Calvez, J. Dorandeu, Y. Faugère, F. Mertz, and P. Schaeffer, 2000: Surface Moyenne Océanique: Support scientifique à la mission altimétrique Jason-1, et à une mission micro-satellite altimétrique. Contrat SSALTO 2945 - Lot 2 A.1, CLS Progress Rep. CLS/DOS/NT/00.313, 40 pp.

Hughes, C. W., and J. R. Bingham, 2008: An oceanographer's guide to GOCE and the geoid. Ocean Sci. Discuss., 4, 15-29, doi:10.5194/os-4-15-2008.

Janjić, T., J. Schröter, R. Savcenko, W. Bosch, A. Albertella, R. Rummel, and O. Klatt, 2012: Impact of combining GRACE and GOCE gravity data on ocean circulation estimates. Ocean Sci., 8, 65-79, doi:10.5194/os-8-65-2012.

Jekeli, C., 1981: Alternative methods to smooth the Earth's gravity field. Rep. 327, Dept. of Civil and Environmental Engineering and Geodetic Science, The Ohio State University, 48 pp.

Jin, S. G., D. P. Chambers, and B. D. Tapley, 2010: Hydrological and oceanic effects on polar motion from GRACE and models J. Geophys. Res., 115, B02403, doi:10.1029/2009JB006635.

_ L. L. Jhang, and B. D. Tapley, 2011: The understanding of length-of-day variations from satellite gravity and laser ranging measurements. Geophys. J. Int., 184, 651-660, doi:10.1111/ j.1365-246X.2010.04869.x.

_- T. van Dam, and S. Wdowinski, 2013: Observing and understanding the Earth system variations from space geodesy. J. Geodyn., 72, 1-10, doi:10.1016/j.jog.2013.08.001.

Knudsen, P., R. Bingham, O. Andersen, and M. H. Rio, 2011: A global mean dynamic topography and ocean circulation estimation using a preliminary GOCE gravity model. J. Geod., 85, 861-879, doi:10.1007/s00190-011-0485-8.

Liu, Y., Y. Yuan, J. Su, and J. Jiang, 2000: Circulation in the South China Sea in summer of 1998. Chin. Sci. Bull., 45, 1648-1655, doi:10.1007/BF02898979.

_- R. H. Weisberg, and Y. Yuan, 2008: Patterns of upper layer circulation variability in the South China Sea from satellite altimetry using the self-organizing map. Acta Oceanol. Sin., 27 (Suppl.), 129-144.

,,-- S. Vignudelli, L. Roblou, and C. Merz, 2012: Comparison of the X-TRACK altimetry estimated currents with moored ADCP and HF radar observations on the West Florida Shelf. Adv. Space Res., 50, 1085-1098, doi:10.1016/ j.asr.2011.09.012.

Losch, M., and J. Schröter, 2004: Estimating the circulation from hydrography and satellite altimetry in the Southern Ocean: Limitations imposed by the current geoid models. Deep-Sea Res. I, 51, 1131-1143, doi:10.1016/j.dsr.2004.02.012.
Lumpkin, R., and M. Pazos, 2007: Measuring surface currents with Surface Velocity Program drifters: The instrument, its data, and some recent results. Lagrangian Analysis and Prediction of Coastal and Ocean Dynamics, A. Griffa et al., Eds., Cambridge University Press, 39-67.

and G. C. Johnson, 2013: Global ocean surface velocities from drifters: Mean, variance, El Niño-Southern Oscillation response, and seasonal cycle. J. Geophys. Res. Oceans, 118, 2992-3006, doi:10.1002/jgrc.20210.

Mayer-Gürr, T., A. Eicker, E. Kurtenbach, and K. H. Ilk, 2010: ITG-GRACE: Global static and temporal gravity field models from GRACE data. System Earth via Geodetic-Geophysical Space Techniques, F. Flechtner et al., Eds., Advanced Technologies in Earth Sciences, Springer, 159-168.

Niiler, P. P., 2001: The world ocean surface circulation. Ocean Circulation and Climate, G. Siedler, Eds., International Geophysics Series, Vol. 77, Academic Press, 193-204.

— , and J. D. Paduan, 1995: Wind-driven motions in the northeast Pacific as measured by Lagrangian drifters. J. Phys. Oceanogr., 25, 2819-2830, doi:10.1175/1520-0485(1995)025<2819: WDMITN $>2.0 . \mathrm{CO} ; 2$.

Pail, R., and Coauthors, 2011: First GOCE gravity field models derived by three different approaches. J. Geod., 85, 819-843, doi:10.1007/s00190-011-0467-x.

Pazan, S. E., and P. P. Niiler, 2001: Recovery of near-surface velocity from undrogued drifters. J. Atmos. Oceanic Technol., 18, 476-489, doi:10.1175/1520-0426(2001)018<0476: RONSVF $>2.0 . \mathrm{CO} ; 2$.

Ralph, E. A., and P. P. Niiler, 1999: Wind-driven currents in the tropical Pacific. J. Phys. Oceanogr., 29, 2121-2129, doi:10.1175/ 1520-0485(1999)029<2121:WDCITT>2.0.CO;2.

Rio, M. H., S. Guinehut, and G. Larnicol, 2011: New CNES-CLS09 global mean dynamic topography computed from the combination of GRACE data, altimetry, and in situ measurements. J. Geophys. Res., 116, C07018, doi:10.1029/2010JC006505.

Sánchez-Reales, J., M. Vigo, S. G. Jin, and B. Chao, 2012: Global surface geostrophic currents of ocean derived from satellite altimetry and GOCE geoid. Mar. Geod., 35 (Supp.), 175-189, doi:10.1080/01490419.2012.718696.

Stammer, D., A. Köhl, and C. Wunsch, 2007: Impact of accurate geoid fields on estimates of the ocean circulation. J. Atmos. Oceanic Technol., 24, 1464-1478, doi:10.1175/JTECH2044.1.

Vignudelli, S., A. G. Kostianoy, P. Cipollini, and J. Benveniste, Eds., 2011: Coastal Altimetry. Springer, 566 pp.

Wahr, J., M. Molenaar, and F. Bryan, 1998: Time variability of the Earth's gravity field: Hydrological and oceanic effects and their possible detection using GRACE. J. Geophys. Res., 103, 30 205-30229, doi:10.1029/98JB02844.

Yuan, Y., A. Kaneko, J. Su, X. Zhu, Y. Liu, N. Gohda, and H. Chen, 1998: The Kuroshio east of Taiwan and in the East China Sea and the currents east of Ryukyu Islands during early summer of 1996.J. Oceanogr., 54, 217-226, doi:10.1007/ BF02751697.

Zhou, D., Y.-B. Liang, C.-K. Zeng, and C. K. Tseng, Eds., 1994: Oceanology of China Seas. Vols. 1 and 2, Kluwer Academic Publishers, 579 pp. 